\title{
q-Hardy-Littlewood-Type Maximal Operator with Weight Related to Fermionic p-Adic q-Integral on $Z_{p}$
}

\author{
Erdoğan Şen ${ }^{1}$, Mehmet Acikgoz ${ }^{2}$, Serkan Araci ${ }^{3, *}$ \\ ${ }^{1}$ Department of Mathematics, Faculty of Science and Letters, Namik Kemal University, Tekirdağ, Turkey \\ ${ }^{2}$ Department of Mathematics, Faculty of Science and Arts, University of Gaziantep, Gaziantep, Turkey \\ ${ }^{3}$ Atatürk Street, 31290 Hatay, TURKEY \\ *Corresponding author: mtsrkn@hotmail.com
}

Received August 14, 2013; Revised September 17, 2013; Accepted September 25, 2013

\begin{abstract}
The q-extension of Hardy-littlewood-type maximal operator in accordance with q-Volkenborn integral in the $p$-adic integer ring was recently studied [11]. A generalization of Jang's results was given by Araci and Acikgoz [1]. By the same motivation of their papers, we aim to give the definition of the weighted $q$-Hardylittlewood-type maximal operator by means of fermionic p-adic q-invariant distribution on $\mathrm{Z}_{\mathrm{p}}$. Finally, we derive some interesting properties involving this-type maximal operator.
\end{abstract}

Keywords: fermionic p-adic q-integral on $Z_{p}$, hardy-littlewood theorem, p-adic analysis, $q$-analysis

Cite This Article: Erdoğan Şen, Mehmet Acikgoz, and Serkan Araci, "q-Hardy-Littlewood-Type Maximal Operator with Weight Related to Fermionic p-Adic q-Integral on $Z_{\mathrm{p}}$." Turkish Journal of Analysis and Number Theory 1, no. 1 (2013): 4-8. doi: 10.12691/tjant-1-1-2.

\section{Introduction}

The concept of $\mathrm{p}$-adic numbers was originally invented by Kurt Hensel who is German mathematician, around the end of the nineteenth century [12]. In spite of their being already one hundred years old, these numbers are still today enveloped in an aura of mystery within the scientific community and also play a vital and important role in mathematics.

The fermionic $\mathrm{p}$-adic $\mathrm{q}$-integral in the p-adic integer ring was originally constructed by $\operatorname{Kim}[2,6]$ who introduced Lebesgue-Radon-Nikodym Theorem with respect to fermionic $\mathrm{p}$-adic $\mathrm{q}$-integral on $\mathrm{Z}_{p}$. The fermionic $\mathrm{p}$-adic q-integral on $\mathrm{Z}_{p}$ is used in mathematical physics for example the functional equation of the q-zeta function, the q-stirling numbers and q-mahler theory of integration with respect to the ring $Z_{p}$ together with Iwasawa's p-adic q-L function.

In [11], Jang defined q-extension of Hardy-Littlewoodtype maximal operator by means of q-Volkenborn integral on $Z_{p}$. Afterwards, in [1], Araci and Acikgoz added a weight into Jang's q-Hardy-Littlewood-type maximal operator and derived some interesting properties by means of Kim's p-adic q-integral on $\mathrm{Z}_{p}$. Now also, we shall consider weighted q-Hardy-Littlewood-type maximal operator on the fermionic p-adic q-integral on $\mathrm{Z}_{p}$. Moreover, we shall analyse q-Hardy-Littlewood-type maximal operator via the fermionic $\mathrm{p}$-adic $\mathrm{q}$-integral on $\mathrm{Z}_{p}$.
Assume that $\mathrm{p}$ be an odd prime number. Let $\mathrm{Q}_{p}$ be the field of $\mathrm{p}$-adic rational numbers and let $\mathrm{C}_{p}$ be the completion of algebraic closure of $\mathrm{Q}_{p}$.

Thus,

$$
\mathrm{Q}_{p}=\left\{x=\sum_{n=-k}^{\infty} a_{n} p^{n}: 0 \leq a_{n}<p\right\} .
$$

Then $\mathrm{Z}_{p}$ is an integral domain to be

$$
\mathrm{Z}_{p}=\left\{x=\sum_{n=0}^{\infty} a_{n} p^{n}: 0 \leq a_{n} \leq p-1\right\},
$$

or

$$
\mathrm{Z}_{p}=\left\{x \in \mathrm{Q}_{p}:|x|_{p} \leq 1\right\} .
$$

In this paper, we assume that $q \in \mathrm{C}_{p}$ with $|1-q|_{p}<1$ as an indeterminate.

The $\mathrm{p}$-adic absolute value $\|_{p}$, is normally defined by

$$
|x|_{p}=\frac{1}{p^{r}}
$$

where $x=p^{r} \frac{s}{t}$ with $(p, s)=(p, t)=(s, t)=1$ and $r \in \mathrm{Q}$.

A $p$-adic Banach space $B$ is a $\mathrm{Q}_{\mathrm{p}}$-vector space with a lattice $B^{0}\left(\mathrm{Z}_{\mathrm{p}}\right.$-module) separated and complete for $\mathrm{p}$-adic topology, ie.,

$$
B^{0} \simeq \varliminf_{n \in \mathrm{N}} B^{0} / p^{n} B^{0} .
$$


For all $x \in B$, there exists $n \in Z$, such that $x \in p^{n} B^{0}$. Define

$$
v_{B}(x)=\sup _{n \in \mathrm{N} \cup\{+\infty\}}\left\{n: x \in p^{n} B^{0}\right\} .
$$

It satisfies the following properties:

$$
\begin{aligned}
v_{B}(x+y) & \geq \min \left(v_{B}(x), v_{B}(y)\right), \\
v_{B}(\beta x) & =v_{p}(\beta)+v_{B}(x), \text { if } \beta \in \mathrm{Q}_{p} .
\end{aligned}
$$

Then, $\|x\|_{B}=p^{-v_{B}(x)}$ defines a norm on $B$, such that $B$ is complete for \|\|$_{B}$ and $B^{0}$ is the unit ball.

A measure on $\mathrm{Z}_{p}$ with values in a p-adic Banach space $B$ is a continuous linear map

$$
f \mapsto \int f(x) \mu=\int_{\mathrm{Z}_{p}} f(x) \mu(x)
$$

from $C^{0}\left(\mathrm{Z}_{p}, \mathrm{C}_{p}\right)$, (continuous function on $\mathrm{Z}_{p}$ ) to $B$. We know that the set of locally constant functions from $\mathrm{Z}_{p}$ to $\mathrm{Q}_{p}$ is dense in $C^{0}\left(\mathrm{Z}_{p}, \mathrm{C}_{p}\right)$ so.

Explicitly, for all $f \in C^{0}\left(\mathrm{Z}_{p}, \mathrm{C}_{p}\right)$, the locally constant functions

$$
f_{n}=\sum_{i=0}^{p^{n}-1} f(i) 1_{i+p^{n} \mathrm{Z}_{p}} \rightarrow f \text { in } C^{0} .
$$

Now if $\mu \in \mathrm{D}_{0}\left(\mathrm{Z}_{p}, \mathrm{Q}_{p}\right)$, set $\mu\left(i+p^{n} \mathrm{Z}_{p}\right)=\int_{\mathrm{Z}_{p}{ }^{i+p^{n}} \mathrm{Z}_{p}} \mu$. Then $\int_{Z_{p}} f \mu$ is given by the following Riemann sums

$$
\int_{\mathrm{Z}_{p}} f \mu=\lim _{n \rightarrow \infty} \sum_{i=0}^{p^{n}-1} f(i) \mu\left(i+p^{n} \mathrm{Z}_{p}\right) .
$$

T. Kim defined $\mu_{-q}$ as follows:

$$
\mu_{-q}\left(\xi+d p^{n} \mathrm{Z}_{p}\right)=\frac{(-q)^{\xi}}{\left[d p^{n}\right]_{-q}}
$$

and this can be extended to a distribution on $Z_{p}$. This distribution yields an integral in the case $d=1$.

So, q-Volkenborn integral was defined by T. Kim as follows:

$$
\begin{aligned}
I_{-q}(f) & =\int_{\mathrm{Z}_{p}} f(\xi) d \mu_{q}(\xi) \\
& =\lim _{n \rightarrow \infty} \frac{1}{\left[p^{n}\right]_{-q}} \sum_{\xi=0}^{p^{n}-1}(-1)^{\xi} f(\xi) q^{\xi}
\end{aligned}
$$

where $[x]_{q}$ is a q-extension of $x$ which is defined by

$$
[x]_{q}=\frac{1-q^{x}}{1-q} .
$$

Note that $\lim _{q \rightarrow 1}[x]_{q}=x$ cf. $[1,2,4,5,6,7,11]$.

Let $d$ be a fixed positive integer with $(p, d)=1$. We now set

$$
\begin{aligned}
X & =X_{d}=\underset{n}{\lim } \mathrm{Z} / d p^{n} \mathrm{Z}, \\
X_{1}= & \mathrm{Z}_{p}, \\
X^{*}= & \underset{\substack{0<a<d p \\
(a, p)=1}}{\cup} a+d p \mathrm{Z}_{p}, \\
a+d p^{n} \mathrm{Z}_{p}= & \left\{x \in X \mid x \equiv a\left(\bmod p^{n}\right)\right\},
\end{aligned}
$$

where $a \in \mathrm{Z}$ satisfies the condition $0 \leq a<d p^{n}$. For $f \in U D\left(\mathrm{Z}_{p}, \mathrm{C}_{p}\right)$,

$$
\int_{Z_{p}} f(x) d \mu_{-q}(x)=\int_{X} f(x) d \mu_{-q}(x) \text { see [10] }
$$

By means of q-Volkenborn integral, we consider below strongly p-adic q-invariant distribution $\mu_{-q}$ on $\mathrm{Z}_{p}$ in the form

$$
\left|\begin{array}{l}
{\left[p^{n}\right]_{-q} \mu_{-q}\left(a+p^{n} Z_{p}\right)} \\
-\left[p^{n+1}\right]_{-q} \mu_{-q}\left(a+p^{n+1} Z_{p}\right)
\end{array}\right|<\delta_{n}
$$

where $\delta_{n} \rightarrow 0$ as $n \rightarrow \infty$ and $\delta_{n}$ is independent of $a$. Let $f \in U D\left(\mathrm{Z}_{p}, \mathrm{C}_{p}\right)$, for any $a \in \mathrm{Z}_{p}$, we assume that the weight function $\omega(x)$ is defined by $\omega(x)=\omega^{x}$ where $\omega \in \mathrm{C}_{p}$ with $|1-\omega|_{p}<1$. We define the weighted measure on $\mathrm{Z}_{p}$ as follows:

$$
\mu_{f,-q}^{(\omega)}\left(a+p^{n} \mathrm{Z}_{p}\right)=\int_{a+p^{n} \mathrm{Z}_{p}} \omega^{\xi} f(\xi) d \mu_{-q}(\xi)
$$

where the integral is the fermionic p-adic q-integral on $Z_{p}$. From (1.2), we note that $\mu_{f,-q}^{(\omega)}$ is a strongly weighted measure on $Z_{p}$. Namely,

$$
\begin{aligned}
& \left|\left[p^{n}\right]_{-q} \mu_{f,-q}^{(\omega)}\left(a+p^{n} \mathrm{Z}_{p}\right)-\left[p^{n+1}\right]_{-q} \mu_{f,-q}^{(\omega)}\left(a+p^{n+1} \mathrm{Z}_{p}\right)\right|_{p} \\
= & \mid \sum_{x=0}^{p^{n}-1}(-1)^{x} \omega^{x} f(x) q^{x}-\sum_{x=0}^{\left.p^{n}(-1)^{x} \omega^{x} f(x) q^{x}\right|_{p}} \\
\leq & \left.\left|\frac{f\left(p^{n}\right)(-1)^{p^{n}} \omega^{p^{n}} q^{p^{n}}}{p^{n}}\right| p^{n}\right|_{p} \\
\leq & C p^{-n}
\end{aligned}
$$

Thus, we get the following proposition.

Proposition 1. For $f, g \in U D\left(\mathrm{Z}_{p}, \mathrm{C}_{p}\right)$, then, we have 


$$
\begin{aligned}
& \mu_{\alpha f+\beta g,-q}^{(\omega)}\left(a+p^{n} Z_{p}\right) \\
= & \alpha \mu_{f,-q}^{(\omega)}\left(a+p^{n} Z_{p}\right)+\beta \mu_{g,-q}^{(\omega)}\left(a+p^{n} Z_{p}\right) .
\end{aligned}
$$

where $\alpha, \beta$ are positive constants. Also, we have

$$
\left|\begin{array}{c}
{\left[p^{n}\right]_{-q} \mu_{f,-q}^{(\omega)}\left(a+p^{n} \mathrm{Z}_{p}\right)} \\
-\left[p^{n+1}\right]_{-q} \mu_{f,-q}^{(\omega)}\left(a+p^{n+1} \mathrm{Z}_{p}\right)
\end{array}\right| \leq C p^{-n}
$$

where $C$ is positive constant.

Let $\mathbf{P}_{q}(x) \in \mathrm{C}_{p}\left[[x]_{q}\right]$ be an arbitrary q-polynomial. Now also, we indicate that $\mu_{\mathrm{P},-q}^{(\omega)}$ is a strongly weighted fermionic $\mathrm{p}$-adic q-invariant measure on $\mathrm{Z}_{p}$. Without a loss of generality, it is sufficient to evidence the statement for $\mathbf{P}(x)=[x]_{q}^{k}$.

$$
\begin{aligned}
& \mu_{\mathrm{P},-q}^{(\omega)}\left(a+p^{n} \mathrm{Z}_{p}\right) \\
= & \lim _{m \rightarrow \infty} \frac{1}{\left[p^{m}\right]_{-q}} \sum_{i=0}^{p^{m-n}-1} w^{a+i p^{n}}\left[a+i p^{n}\right]_{q}^{k}(-q)^{a+i p^{n}}
\end{aligned}
$$

where

$$
\begin{aligned}
& {\left[a+i p^{n}\right]_{q}^{k} } \\
= & \sum_{j=0}^{k}\left(\begin{array}{c}
k \\
j
\end{array}\right)[a]_{q}^{k-j} q^{a j}\left[p^{n}\right]_{q}^{j}[i]_{q}^{j} p^{n} \\
= & {[a]_{q}^{k}+k[a]_{q}^{k-1} q^{a}\left[p^{n}\right]_{q}[i]_{q} p^{n} } \\
& +\ldots+q^{a k}\left[p^{n}\right]_{q}^{k}[i]_{q}^{k} p^{n}
\end{aligned}
$$

and

$$
\begin{aligned}
& w^{a+i p^{n}}=w^{a} \sum_{l=0}^{i p^{n}}\left(\begin{array}{c}
i p^{n} \\
l
\end{array}\right)(w-1)^{l} \\
& \equiv w^{a}\left(\bmod p^{n}\right) .
\end{aligned}
$$

By (1.5), we have

$$
\begin{aligned}
& (-q)^{a+i p^{n}} \\
= & (-q)^{a} \sum_{l=0}^{i p^{n}}\left(\begin{array}{c}
i p^{n} \\
l
\end{array}\right)(-1)^{l}(q+1)^{l} \\
\equiv & (-q)^{a}\left(\bmod p^{n}\right) .
\end{aligned}
$$

By (1.3), (1.4), (1.5) and (1.6), we have the following

$$
\begin{aligned}
& \mu_{\mathrm{P},-q}^{(\omega)}\left(a+p^{n} \mathrm{Z}_{p}\right) \\
\equiv & (-1)^{a} \omega^{a} q^{a}[a]_{q}^{k}\left(\bmod p^{n}\right) \\
\equiv & (-1)^{a} \omega^{a} q^{a} \mathrm{P}(a)\left(\bmod p^{n}\right) .
\end{aligned}
$$

For $\quad x \in Z_{p} \quad, \quad$ let $\quad x \equiv x_{n}\left(\bmod p^{n}\right) \quad$ and $x \equiv x_{n+1}\left(\bmod p^{n+1}\right) \quad, \quad$ where $\quad x_{n}, \quad x_{n+1} \in \mathrm{Z} \quad$ with $0 \leq x_{n}<p^{n}$ and $0 \leq x_{n+1}<p^{n+1}$

Then, we procure the following

$$
\left|\begin{array}{l}
{\left[p^{n}\right]_{-q} \mu_{\mathrm{P},-q}^{(\omega)}\left(a+p^{n} \mathrm{Z}_{p}\right)} \\
-\left[p^{n+1}\right]_{-q} \mu_{\mathrm{P},-q}^{(\omega)}\left(a+p^{n+1} \mathrm{Z}_{p}\right)
\end{array}\right| \leq C p^{-n},
$$

where $C$ is positive constant and $n>>0$.

Let $U D\left(\mathrm{Z}_{p}, \mathrm{C}_{p}\right)$ be the space of uniformly differentiable functions on $Z_{p}$ with sup-norm

$$
\|f\|_{\infty}=\sup _{x \in Z_{p}}|f(x)|_{p} .
$$

The difference quotient $\Delta_{1} f$ of $f$ is the function of two variables given by

$$
\Delta_{1} f(m, x)=\frac{f(x+m)-f(x)}{m}
$$

for all $x, m \in \mathrm{Z}_{p}, m \neq 0$

A function $f: Z_{p} \rightarrow \mathrm{C}_{p}$ is said to be a Lipschitz function if there exists a constant $M>0$ (the Lipschitz constant of $f$ ) such that

$$
\left|\Delta_{1} f(m, x)\right| \leq M \text { for all } m \in Z_{p} \backslash\{0\} \text { and } x \in Z_{p} .
$$

The $\mathrm{C}_{p}$ linear space consisting of all Lipschitz function is denoted by $\operatorname{Lip}\left(\mathrm{Z}_{p}, \mathrm{C}_{p}\right)$. This space is a Banach space with the respect to the norm $\|f\|_{1}=\|f\|_{\infty} \vee\left\|\Delta_{1} f\right\|_{\infty}$ (for more information, see [3-9]). The objective of this paper is to introduce weighted q-Hardy Littlewood-type maximal operator on the fermionic p-adic q-integral on $\mathrm{Z}_{p}$. Also, we show that the boundedness of the weighted q-Hardylittlewood-type maximal operator in the p-adic integer ring.

\section{The Weighted q-Hardy-Littlewood- Type Maximal Operator}

In view of (1.2) and the definition of fermionic p-adic qintegral on $\mathrm{Z}_{p}$, we now consider the following theorem.

Theorem 1. Let $\mu_{-q}^{(w)}$ be a strongly fermionic $p$-adic $q$ invariant on $\mathrm{Z}_{p}$ and $f \in U D\left(\mathrm{Z}_{p}, \mathrm{C}_{p}\right)$. Then for any $n \in \mathrm{Z}$ and any $\xi \in \mathrm{Z}_{p}$, we have

$$
\begin{aligned}
& \int_{a+p^{n} \mathrm{Z}_{p}} \omega^{\xi} f(\xi)(-q)^{-\xi} d \mu_{-q}(\xi) \\
\text { (1) }= & \frac{(-1)^{a} \omega^{a}}{\left[p^{n}\right]_{-q}} \int_{\mathrm{Z}_{p}} \omega^{\xi} f\left(a+p^{n} \xi\right)(-q)^{-p^{n} \xi} d \mu_{-q} p^{n}
\end{aligned}
$$


(2) $\int_{a+p^{n} \mathrm{Z}_{p}} \omega^{\xi} d \mu_{-q}(\xi)=\frac{\omega^{a}(-q)^{a}}{\left[p^{n}\right]_{-q}} \frac{2}{1+\omega^{p^{n}}{ }_{q^{p^{n}}}}$

Proof. (1) By using (1.1) and (1.2), we see the following applications:

$$
\begin{aligned}
& \int_{a+p^{n} \mathrm{Z}_{p}} \omega^{\xi} f(\xi)(-q)^{-\xi} d \mu_{-q}(\xi) \\
& =\lim _{m \rightarrow \infty} \frac{1}{\left[p^{m+n}\right]_{-q}} \sum_{\xi=0}^{p^{m}-1}\left[\begin{array}{l}
\omega^{a+p^{n} \xi} f\left(a+p^{n} \xi\right) \\
\times(-q)^{-\left(a+p^{n} \xi\right)} \\
\times q^{a+p^{n} \xi}(-1)^{a+p^{n} \xi}
\end{array}\right] \\
& =(-1)^{a} \omega^{a} \lim _{m \rightarrow \infty}\left[\begin{array}{c}
\frac{1}{\left[p^{m}\right]_{-q} p^{n}\left[p^{n}\right]_{-q}} \times \sum_{\xi=0}^{p^{m}-1} \omega^{\xi}(-q)^{-p^{n} \xi} \\
\times f\left(a+p^{n} \xi\right)\left(-q^{p^{n}}\right)^{\xi}
\end{array}\right] \\
& =\frac{(-1)^{a} \omega^{a}}{\left[p^{n}\right]_{-q}} \int_{\mathrm{Z}_{p}}\left[\begin{array}{c}
\omega^{\xi} f\left(a+p^{n} \xi\right) \\
\times(-q)^{-p^{n} \xi} d \mu_{-q}^{p^{n}}(\xi)
\end{array}\right] \text {. }
\end{aligned}
$$

(2) By the same method of (1), then, we easily derive the following

$$
\begin{aligned}
& \int_{a+p^{n} \mathrm{Z}_{p}} \omega^{\xi} d \mu_{-q}(\xi) \\
= & \lim _{m \rightarrow \infty} \frac{1}{\left[p^{m+n}\right]_{-q}} \sum_{\xi=0}^{p^{m}-1} \omega^{a+\xi p^{n}}(-q)^{a+\xi p^{n}} \\
= & \frac{\omega^{a}(-q)^{a}}{\left[p^{n}\right]_{-q}} \lim _{m \rightarrow \infty} \frac{1}{\left[p^{m}\right]_{-q} p^{n}} \sum_{\xi=0}^{p^{m}-1}\left(\omega^{p^{n}}\right)^{\xi}\left(-q^{p^{n}}\right)^{\xi} \\
= & \frac{\omega^{a}(-q)^{a}}{\left[p^{n}\right]_{-q}} \lim _{m \rightarrow \infty} \frac{1+\left(\omega^{p^{n}} q^{p^{n}}\right)^{p^{m}}}{1+\omega^{p^{n}} q^{p^{n}}} \\
= & \frac{\omega^{a}(-q)^{a}}{\left[p^{n}\right]_{-q}} \frac{2}{1+\omega^{p^{n}} q^{p^{n}}}
\end{aligned}
$$

Since $\lim _{m \rightarrow \infty} q^{p^{m}}=1$ for $|1-q|_{p}<1$, our assertion follows.

We are now ready to introduce the definition of the weighted q-Hardy-littlewood-type maximal operator related to fermionic $\mathrm{p}$-adic $\mathrm{q}$-integral on $\mathrm{Z}_{p}$ with a strong fermionic p-adic q-invariant distribution $\mu_{-q}$ in the p-adic integer ring.
Definition 1. Let $\mu_{-q}^{(\omega)}$ be a strongly fermionic p-adic $q$ invariant distribution on $\mathrm{Z}_{p}$ and $f \in U D\left(\mathrm{Z}_{p}, \mathrm{C}_{p}\right)$. Then, $q$-Hardy-littlewood-type maximal operator with weight related to fermionic p-adic q-integral on $a+p^{n} \mathrm{Z}_{p}$ is defined as

$$
\begin{aligned}
& \mathbf{M}_{p, q}^{(\omega)} f(a) \\
= & \sup _{n \in \mathrm{Z}} \frac{1}{\mu_{1,-q}^{(w)}\left(\xi+p^{n} \mathrm{Z}_{p}\right)} \int_{a+p^{n} \mathrm{Z}_{p}} \omega^{\xi}(-q)^{-\xi} f(\xi) d \mu_{-q}(\xi)
\end{aligned}
$$

for all $a \in \mathrm{Z}_{p}$.

We recall that famous Hardy-littlewood maximal operator $\mathbf{M}_{\mu}$, which is defined by

$$
\mathbf{M}_{\mu} f(a)=\sup _{a \in Q} \frac{1}{\mu(Q)} \int_{Q}|f(x)| d \mu(x),
$$

where $f: \mathrm{R}^{k} \rightarrow \mathrm{R}^{k}$ is a locally bounded Lebesgue measurable function, $\mu$ is a Lebesgue measure on $(-\infty, \infty)$ and the supremum is taken over all cubes $Q$ which are parallel to the coordinate axes. Note that the boundedness of the Hardy-Littlewood maximal operator serves as one of the most important tools used in the investigation of the properties of variable exponent spaces (see [11]). The essential aim of Theorem 1 is to deal mainly with the weighted q-extension of the classical Hardy-Littlewood maximal operator in the space of p-adic Lipschitz functions on $\mathrm{Z}_{p}$ and to find the boundedness of them. By means of Definition 1, then, we state the following theorem.

Theorem 2. Let $f \in U D\left(\mathrm{Z}_{p}, \mathrm{C}_{p}\right)$ and $x \in \mathrm{Z}_{p}$, we get

$$
\begin{aligned}
\mathbf{M}_{p, q}^{(\omega)} f(a)= & \frac{(-1)^{a}}{2 q^{a}} \sup \left(\begin{array}{c}
1+\omega^{p^{n} q^{p^{n}}} \\
n \in \mathrm{Z}
\end{array}\right) \\
& \int_{\mathrm{Z}_{p}} \omega^{\xi} f\left(x+p^{n} \xi\right)(-q)^{-p^{n} \xi} d \mu_{-q} p^{n}
\end{aligned}
$$

(2)

$$
\left|\mathbf{M}_{p, q}^{(\omega)} f(a)\right|_{p}
$$

$$
\leq\left|\frac{(-1)^{a}}{2 q^{a}}\right|_{p} \sup _{n \in Z}\left|1+\omega^{p^{n}} q^{p^{n}}\right|_{p}\|f\|_{1}\left\|\left(\frac{-q^{p^{n}}}{\omega}\right)^{-(.)}\right\|_{L^{1}}
$$

where $\left\|\left(\frac{-q^{p^{n}}}{\omega}\right)^{-(.)}\right\|_{L^{1}}=\int_{\mathrm{Z}_{p}}\left(\frac{-q^{p^{n}}}{\omega}\right)^{-\xi} d \mu_{-q^{p^{n}}}(\xi)$

Proof. (1) Because of Theorem 1 and Definition 1, we see

$$
\begin{aligned}
\mathbf{M}_{p, q}^{(\omega)} f(a)= & \sup _{n \in \mathrm{Z}} \frac{1}{\mu_{1,-q}^{(\omega)}\left(\xi+p^{n} \mathrm{Z}_{p}\right)} \\
& \int_{a+p^{n} \mathrm{Z}_{p}} \omega^{\xi}(-q)^{-\xi} f(\xi) d \mu_{-q}(\xi)
\end{aligned}
$$




$$
\begin{aligned}
= & \frac{(-1)^{a}}{2 q^{a}} \sup \left(1+\omega^{p^{n} q^{p^{n}}}\right) \\
& \int_{\mathrm{Z}_{p}} \omega^{\xi} f\left(x+p^{n} \xi\right)(-q)^{-p^{n} \xi} d \mu_{-q} p^{n}
\end{aligned}
$$

(2) On account of (1), we can derive the following

$$
\begin{aligned}
& \left|\mathbf{M}_{p, q}^{(\omega)} f(a)\right|_{p} \\
& =\left|\begin{array}{l}
\frac{(-1)^{a}}{2 q^{a}} \sup _{n \in \mathrm{Z}}\left(1+\omega^{p^{n}} q^{p^{n}}\right) \\
\int_{\mathrm{Z}_{p}} \omega^{\xi} f\left(x+p^{n} \xi\right)(-q)^{-p^{n} \xi} d \mu_{-q} p^{n}(\xi)
\end{array}\right|
\end{aligned}
$$

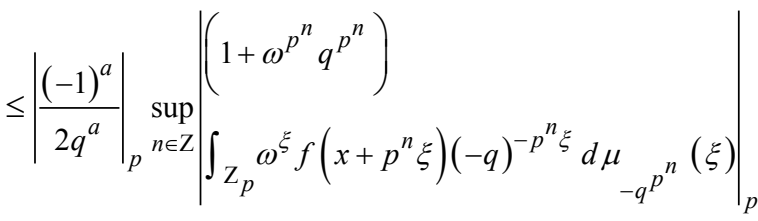

$$
\begin{aligned}
& \leq\left|\frac{(-1)^{a}}{2 q^{a}}\right|_{p} \sup _{n \in \mathrm{Z}}\left|1+\omega^{p^{n}} q^{p^{n}}\right|_{p} \\
& \int_{\mathrm{Z}_{p}}\left|f\left(a+p^{n} \xi\right)\right|_{p}\left|\left(\frac{-q^{p^{n}}}{\omega}\right)^{-\xi}\right|_{p} d \mu_{-q} p^{n}(\xi) \\
& \leq\left|\frac{(-1)^{a}}{2 q^{a}}\right|_{p} \sup _{n \in \mathrm{Z}}\left|1+\omega^{p^{n}} q^{p^{n}}\right|_{p}\|f\|_{1} \\
& \int_{\mathrm{Z}_{p}}\left|\left(\frac{-q^{p^{n}}}{\omega}\right)^{-\xi}\right|_{p} d \mu p^{n}(\xi) \\
& =\left|\frac{(-1)^{a}}{2 q^{a}}\right|_{p} \sup _{n \in \mathrm{Z}}\left|1+\omega^{p^{n}} q^{p^{n}}\right|_{p}\|f\|_{1} \mid\left(\frac{-q^{p^{n}}}{\omega}\right)^{-(.)} \|_{L^{1}} .
\end{aligned}
$$

Thus, we complete the proof of theorem.

We note that Theorem 2 (2) shows the supnorminequality for the q-Hardy-Littlewood-type maximal operator with weight on $Z_{p}$, on the other hand, Theorem 2 (2) shows the following inequality

$$
\begin{aligned}
& \left\|\mathbf{M}_{p, q}^{(\omega)} f\right\|_{\infty} \\
= & \sup _{x \in \mathrm{Z}_{p}}\left|\mathbf{M}_{p, q}^{(\omega)} f(x)\right|_{p} \\
\leq & \mathbf{K}\|f\|_{1}\left\|\left(\frac{-q^{p^{n}}}{\omega}\right)^{-(.)}\right\|_{L^{1}}
\end{aligned}
$$

where $\mathbf{K}=\left|\frac{(-1)^{a}}{2 q^{a}}\right|_{p} \sup _{n \in Z}\left|1+\omega^{p^{n}} q^{p^{n}}\right|_{p}$. By the equation (2.2), we get the following Corollary, which is the boundedness for weighted q-Hardy-Littlewood-type maximal operator with weight on $Z_{p}$.

Corollary 1. $\mathbf{M}_{p, q}^{(\omega)}$ is a bounded operator from $U D\left(\mathrm{Z}_{p}, \mathrm{C}_{p}\right)$ into $L^{\infty}\left(\mathrm{Z}_{p}, \mathrm{C}_{p}\right)$, where $L^{\infty}\left(\mathrm{Z}_{p}, \mathrm{C}_{p}\right)$ is the space of all p-adic supnorm-bounded functions with the

$$
\|f\|_{\infty}=\sup _{x \in Z_{p}}|f(x)|_{p}
$$

for all $f \in L^{\infty}\left(\mathrm{Z}_{p}, \mathrm{C}_{p}\right)$.

\section{References}

[1] S. Araci and M. Acikgoz, A note on the weighted $q$-Hardylittlewood-type maximal operator with respect to q-Volkenborn integral in the p-adic integer ring, J. Appl. Math. \& Informatics, Vol. 31(2013), No. 3-4, pp. 365-372.

[2] S. Araci, M. Acikgoz and E. Şen, On the extended Kim's p-adic qdeformed fermionic integrals in the p-adic integer ring, Journal of Number Theory 133 (2013) 3348-3361.

[3] T. Kim, Lebesgue-Radon-Nikodym theorem with respect to fermionic p-adic invariant measure on $\mathrm{Z}_{\mathrm{p}}$, Russ. J. Math. Phys. 19 (2012).

[4] T. Kim, Lebesgue-Radon-Nikodym theorem with respect to fermionic $\mathrm{q}-$ Volkenborn distribution on $\mu_{\mathrm{p}}$, Appl. Math. Comp. 187 (2007), 266-271.

[5] T. Kim, S. D. Kim, D.W. Park, On Uniformly differntiabitity and q-Mahler expansion, Adv. Stud. Contemp. Math. 4 (2001), 35-41.

[6] T. Kim, q-Volkenborn integration, Russian J. Math. Phys. 9 (2002) 288-299.

[7] T. Kim, On a q-analogue of the p-adic log Gamma functions and related integrals, Journal of Number Theory 76 (1999), 320-329.

[8] T. Kim, Note on Dedekind-type DC sums, Advanced Studies in Contemporary Mathematics 18(2) (2009), 249-260.

[9] T. Kim, A note on the weighted Lebesgue-Radon-Nikodym Theorem with respect to p-adic invariant integral on $\mathrm{Z}_{\mathrm{p}}, J$. Appl. Math. \& Informatics, Vol. 30(2012), No. 1, 211-217.

[10] T. Kim, Non-archimedean q-integrals associated with multiple Changhee q-Bernoulli polynomials, Russ. J. Math Phys. 10 (2003) 91-98.

[11] L-C. Jang, On the q-extension of the Hardy-littlewood-type maximal operator related to $\mathrm{q}-$ Volkenborn integral in the p-adic integer ring, Journal of Chungcheon Mathematical Society, Vol. 23, No. 2, June 2010.

[12] K. Hensel, Theorie der Algebraischen Zahlen I. Teubner, Leipzig, 1908.

[13] N. Koblitz, p-adic Numbers, p-adic Analysis and Zeta Functions, Springer-Verlag, New York Inc, 1977. 